\title{
Automated Annotation of Multimedia Audio Data with Affective Labels for Information Management
}

\author{
Ching Hau Chan and Gareth J. F. Jones \\ Centre for Digital Video Processing \& School of Computing \\ Dublin City University, Dublin 9, Ireland \\ email: \{chchan, gjones\}ecomputing.dcu.ie
}

\begin{abstract}
The emergence of digital multimedia systems is creating many new opportunities for rapid access to huge content archives. In order to fully exploit these information sources, the content must be annotated with significant features. An important aspect of human interpretation of multimedia data, which is often overlooked, is the affective dimension. Such information is a potentially useful component for content-based classification and retrieval. Much of the affective information of multimedia content is contained within the audio data stream. Emotional features can be defined in terms of arousal and valence levels. In this study low-level audio features are extracted to calculate arousal and valence levels of multimedia audio streams. These are then mapped onto a set of keywords with predetermined emotional interpretations. Experimental results illustrate the use of this system to assign affective annotation to multimedia data.
\end{abstract}

\section{Introduction}

Computer systems now enable enormous volumes of high quality multimedia content to be distributed across the Internet. In order to most effectively exploit this data, content providers require effective tools for efficient selection and classification of relevant content. A core technology for such information management tools is the automated analysis of the content to index significant features. The content of multimedia data relates both to clearly defined semantic units such as words and visual objects, and to more subjective interpretation, such as the emotions it elicits in those viewing it. Existing work on multimedia content analysis has largely concentrated on recognition of the objective features. A number of current large-scale prototype multimedia digital library systems based on using these features have been developed, for example [1][2]. The feature indexing tools within these systems rely on extraction of low-level feature analysis such as the pitch or frequency spectrum of the audio or the colour distribution and texture in the video. These low-level features can also be used to recognize nonverbal features in multimedia audio such as special effects [3], and can be interpreted in terms of their contribution to the affective labelling of multimedia data [4]. In this paper we describe our system for analysis and interpretation of audio data streams to provide verbal annotation of affect within multimedia documents.

Interpretation of the affective dimension is an important natural component of human classification and retrieval of information. Affective Intelligence is defined in [5] as the communication and management of affective information in human-computer 
interaction. Audio data streams contain significant affective information, recognizing this dimension of multimedia data enables us to label it with emotional metadata tags for use in information management applications. As more and more human tasks are automated by computers, people will have to spend more time interacting with computers. Making sure that their senses are stimulated appropriately is a key element in delivering a more natural communications environment. A good example is how music and sound effects are often used to great effect to convey emotional information in movies and commercials. A current example of the augmentation of verbal labelling to take account of affective interpretation is the Emotive Alert system [6] from the MIT Media Lab. Emotive Alert filters voicemail messages according to various affective axes, including urgency, formality, and happy or sad. Such labels may be used on their own or to complement existing techniques. For example, Emotive Alert and word-level recognition could be combined to realize new ways of browsing for relevant messages. Automatically labelling the significant affective features of multimedia content potentially allows a new dimension for user interaction with multimedia content. For example, you could search for sad or happy sections of a movie, or compare the structure of movies in terms of emotional development. Such methods could also be used to label emotional expressions in discussions or debates, e.g. anger or sadness, alongside the verbal transcriptions currently used to manage this content. Thus a different response may be produced for the same transcribed content depending on their affective context. The system presented in this paper is a step toward labelling the emotional dimension of audio streams using low-level analysis of audio features and their associations with emotional labels. A similar idea is explored for manually assigned metadata in [7], which examines the structure of movies based on the emotional priming of words appearing in "audio descriptions" provided for sight impaired viewers.

This paper is organized as follows: Section 2 introduces affect representation in the context of describing our affect annotation system, and reviews existing work in audio analysis for affect extraction and labelling, Section 3 gives examples of our current experimental investigations, and Section 4 summarizes our conclusions and outlines our ongoing work.

\section{Representation, Extraction and Labelling of Affective States}

Our affect annotation system combines work from fundamental representation of emotional states, with methods to recognize these states in audio content.

\subsection{Dimensional Representation of Affective States}

It is demonstrated in [8][9] that human affective states can be adequately described in terms of three basic underlying dimensions: arousal, valence and control (dominance). Arousal is a continuous response ranging from one extreme of sleep through intermediate states of drowsiness and then alertness through to frenzied excitement. Valence describes the degree of pleasantness-unpleasantness ranging from a "positive" response associated with extreme happiness or ecstasy through to a "negative" response resulting from extreme pain or unhappiness. Control is useful to distinguish between affective 
states with similar arousal and valence, with differing feelings of control or influence over events ranging from total control to no control. From this definition "emotion" not only describes occasional passionate incidents, but rather a person is viewed as being in an emotional state within this three dimensional space at all times.

It has been observed that the control dimension plays only a limited role in extracting affective content from multimedia data [10]. In our work we thus follow the strategy adopted in [4] of measuring only arousal and valence values. Measuring arousal and valence levels across a multimedia document enables us to extract information of its affective content. For example, regions with high arousal are likely to correspond to the "most exciting" segments, and areas of low valence to regions which may provoke a negative response in the viewer. A classic way to combine measurements of arousal and valence is the affect curve. This plots arousal on the y-axis against valence on the $\mathrm{x}$-axis. Each point of this curve corresponds to an affective state. Plotted across a multimedia document, regions of the curve where temporally close points are located in clusters correspond to affective states prevailing in these regions. Figure 3 shows one of our experimental affect curves. Details of the derivation and further interpretation of these affect curves to annotate audio soundtracks are described in the following sections.

\subsection{Extraction of Arousal and Valence from Audio Data}

In order to be able to represent the affective dimension of multimedia audio data, we need to extract arousal and valence information. Methods for doing this for voiced speech in the context of audio-visual recordings are introduced in [4], where the extraction methods are based on a combination of analysis of both audio and visual features. In our current study, we adopt similar extraction methods to those used in [4], but focus only on the audio information stream and analyze all the audio data, including voiced and unvoiced speech, music, special effects and silence. The following subsections outline our methods to extract arousal and valence information.

Arousal Modelling The arousal level is modelled based on the energy of the audio signal. The sampled audio data is first divided into overlapping frames. The short-time energy of an audio signal is defined as,

$$
E_{n}=\frac{1}{N} \sum_{m}[x(m) w(n-m)]^{2}
$$

where $x(m)$ is the sampled audio signal, $n$ is the time index of the short-time energy, and $w(m)$ a rectangular window. If it is assumed that the audio signal changes relatively slowly within a short interval and taking a suitably small frame, $E_{n}$ is calculated for each frame [3]. In our experiments using a frame size of $20 \mathrm{~ms}$ and a sampling frequency of $44.1 \mathrm{kHz}$ with a $2 / 3$ overlap of frames was found to be effective. The energy level can vary significantly between adjacent frames. However, variations in emotion do not occur abruptly in this manner. For example, excitement may increase as the action develops, for instance during the build up to an attack on goal in a soccer match, and then decrease gradually afterwards. In order to more appropriately model overall changes in arousal levels as affective state of the content changes, the measured shorttime energy is convoluted with a long Kaiser window as suggested in [4]. 
Valence Modelling It is noted in [12] that extraction of features to determine valence values is much more difficult than arousal. In our system we currently use pitch to measure the magnitude and sign of valence, as suggested in [4]. We do this by calculating the short-time fundamental frequency based on peak detection from the spectrum of the sound. This is done using an autoregressive model of order 40 as described in [3]. The spectrum calculated using the autoregressive model makes it much easier to extract harmonic peaks in the spectrum, including the fundamental frequency, than one computed directly using a fast Fourier transform. Short-time fundamental frequency values are calculated for the same $20 \mathrm{~ms}$ frames as used to calculate the arousal values. Using this model is important for our system where the data is very varied, and thus a robust method of frequency analysis is required.

Valence varies from a "neutral feeling" towards varying levels of either negative or positive response. The system needs to identify a frequency corresponding to this neutral feeling; points whose pitch value varies from this can then be labelled with a degree of positive or negative valence. The neutral pitch will vary with the audio source type and even between different speakers. There are two issues to be addressed here: identifying the source, and computing a neutral pitch for each source. The measured pitch of each point should then be normalized relative to the appropriate neutral state. This problem is recognized in [4] which is only concerned with the measurement of valence for voiced speak. The approach taken in this method was to assume a fixed neutral pitch for all sources and subtract this from measured values. Due to the greater variation in the sound sources passed to our system and the consequential variation in neutral pitch, we currently take the approach of assuming that the mean pitch value of the signal corresponds to the neutral state and subtract this from all the points in the data stream. We realize that this method assumes that sources will have similar amounts of positive and negative response, which may not be the case for some multimedia sources. We are exploring methods to compute a more accurate source dependent neutral frequency.

The normalized measured pitch values are subject to large changes between adjacent frames, similar to the variation in the short-time power used to measure the arousal, and for the same reason as before, we again smooth the valence function using a long Kaiser window.

\subsection{Verbal Labelling of Affective States}

The interpretation of the arousal and valence values extracted using the audio analysis methods described in the previous section, is much easier if they can be identified with verbal labels corresponding to associated affective states. In order to do this we have developed a method based on results of experiments described in [7][8]. In the study described in [8] a group of subjects were asked to define 151 emotion-denoting keywords in terms of the dimensions of arousal, valence and dominance. Each subject labelled a subset of the keyword set, with each keyword being assessed by an average of 30 subjects. Mean values of the dimension values were then computed across all subjects rating each keyword. The mean values were then transformed linearly to a scale ranging from -1 to +1 with a neutral value of 0 . For example, the keyword "bold" has arousal 0.61 , valence 0.44 and dominance 0.66 , and "nonchalant" has arousal -0.25 , valence 0.07 and dominance 0.11 . These values are of the order that we would expect 
to see based on the definitions of arousal, valence and dominance in Section 2.1. Using the results from [8], we can associate each measured point on an affect curve with the nearest keyword. In order to fit our arousal and valence results onto the same scale as the emotion-denoting keyword the values are scaled across the document into the range -1 to +1 . This once again assumes that the full range of states is suitable for all multimedia data sources, exploring methods of determining affective range within individual documents is the subject of ongoing work.

The final stage of the labelling method is to associate each keyword with an affective state. We do this by utilizing part of the approach to affective labelling based on audio descriptions of movies in [7]. Audio descriptions are carefully scripted spoken annotations added to movies for the visually-impaired. In the gaps between existing dialogue a narrator gives important information regarding on-screen scenes and events, and about characters' actions and appearances. The method described in [7] scans these audio descriptions for words corresponding to visibly manifested emotions. This approach is based on a cognitive theory of emotion proposed in [11]. This theory treats emotions as agents' appraisals of actions, events and objects in their environments, where these are made with respect to their important goals. In terms of this theory, if a character is in an affective state of "delight" it is because something unexpected has happened that assists them in achieving one of their goals. Our system follows the approach of [7] in mapping the recognized affect states to one of the 22 emotion classes proposed in [11]. In [7] the 22 emotions are expanded to a set of 627 emotion keywords using the WordNet ontology. For example, the emotion class "fear" is associated with the keyword "alarmed". When "alarmed" is observed in the audio description, this position in the data is annotated with the emotion "fear".

Our system annotates multimedia audio data with occurrences of the 22 emotion classes by mapping the set of 151 emotion-denoting keywords from [8] to the 22 emotions classes. We found that 50 of the 627 keywords from [7] were present in the list of 151 words; we then manually mapped the remaining 101 words onto the emotion classes using a dictionary to identify the nearest emotion for each word. In operation each point on our automatically extracted audio affect curve is labelled with the nearest of the 151 emotion-denoting keywords and then mapped to the corresponding emotion class.

\section{Experimental Investigation}

In this section we present some examples of content annotation with out affect labelling system. The system has so far been tested with a range of video types, including television entertainment shows and movies. The results shown are an excerpt from a television entertainment show and sections from the movie Enigma.

Excerpt from the show Magic. Figures 1 and 2 show arousal and valence plotted against time for a section from a television show called Magic. The excerpt here is for the magician's bullet trick. The magician fires a bullet which his colleague (apparently!) catches in his mouth. In this scene the magician first describes what will happen during which there is some solitary applause, the bullet is fired and then the watching audience 
applauds. The arousal and valence are low in the first sections and then increase after the firing of the bullet. This is easily explained in terms of increased energy for the arousal graph and higher frequencies for the valence graph when the audience applauds.

The corresponding affect curve plotting the arousal and valence from Figures 1 and 2 is shown in Figure 3. The early sections of this are concentrated around the centre of the plot before moving to the upper right section at the end. Figure 4 shows the verbal affect labels against time assigned based on the path of the affect curve. From this we can see generally negative affect in the early part and more positive emotions, including joy and pride, in the latter section.

Sections from the movie Enigma. The next results are based on the analysis of two 10 minute sections from the movie Enigma. Figures 5 and 6 show the arousal and valence graphs for the first 10 minutes of the movie, with Figures 7 and 8 showing the corresponding affect curve and affect label plots. Figures 9, 10, 11 and 12 show similar plots for a further 10 minute section of the movie beginning at the end of the first hour.

The first 10 minute section opens with orchestral music after which a military officer briefs a meeting of colleagues, after this a discussion takes place including vigorous questioning of one of the officers. From the affect label plot in Figure 8, we can see that the whole section is dominated by negative emotions, the opening sections showing war scenes exhibit fear, distress and remorse, in the middle of the address these disappear and the scene is dominated by pride during the officer's address, finally the scene ends with the fear and shame during the questioning of the officer.

The second 10 minute section shows a scene which opens with a car chase with sound effects of car engines and a siren. The chase ends when the pursing car crashes, and the other car escapes and then hides in a barn. The occupants of the car then set to work with an Enigma machine, but soon become aware that their pursuers are close by. At the end of the scene they are discovered and a conversation begins. The arousal graph in Figure 9 begins with high energy during the car chase which then reduces dramatically once the chase is over. The valence is negative during the car chase and the discovery in the barn, with higher values when it appears that the car has escaped. These results are reflected in the affect curve in Figure 11 which shows large variations in both arousal and valence at different times. The affect label plot in Figure 12 shows early sections of fear, hate and distress, these largely disappear after the car chase when we see a section of relief and admiration, during the search there is tension and suspense before the discovery near the end of scene, during this we see the reemergence of fear and hate, and finally relief when the pursers engage in conversation rather violence.

We notice in all the affect label plots that some emotions, such as reproach and pride, appear regularly and apparently randomly without clear evidence from the audio that these are appropriate labels. We hypothesize that this may be due to an interaction between the kaiser smoothing and position of these labels of the affect curve. We are currently investigating this behaviour.

\section{Conclusions and Further Work}

This paper has demonstrated that we are able to assign reasonable affective labels to multimedia content based on the audio data stream. While the results from this initial 
study are encouraging, we need to develop a larger and independently labelled test corpus to provide clearer quantitative analysis of system performance. We will use this to develop and explore the effectiveness of extended schemes to measure arousal and valence, such as those introduced in [4] which incorporate visual motion activity and density of shot cuts from the video stream as components in the arousal measure, and to explore methods for identifying dominant emotions.

In our ongoing work we are extending our study to compare our automatic audio annotation with the affect labels generated in [7] using the manual audio descriptions. Results of this comparison may lead to methods of effectively combining the alternative annotation schemes to provide richer or more reliable affective labelling. We also plan to explore the use of affect annotation in the automatic comparison of multimedia documents for both retrieval and classification applications. This information might be used to recommend items that a user might like, for example movies with similar structure to those that they have viewed previously.

\section{References}

[1] Smeaton, A.F., Lee, H. and McDonald, K.: Experiences of Creating Four Video Library Collections with the Fischlar System, International Journal on Digital Libraries, 4(10) (2004) 42-44

[2] Hauptmann, A.G., Christel, M.G.: Successful Approaches in the TREC Video Retrieval Evaluations, Proceedings of ACM Multimedia 2004, New York City, ACM (2004) 668-675

[3] Zhang, T., Kuo, C. C. J.: Content-Based Audio Classification and Retrieval for Audiovisual Data Parsing, Kluwer Academic Publishers, (2001)

[4] Hanjalic, A., Xu, L.-Q.: User-Oriented Affective Video Content Analysis, Proceedings of the IEEE Workshop on Content-based Access of Image and Video Libraries (CBAIVL'01), IEEE (2001) 50-57.

[5] Picard, R.W., Cosier, G.: Affective Intelligence - the Missing Link?, BT Technology Journal Vol 14 No 4, (1997)

[6] Inanoglu, Z., Caneel, R.: Emotive Alert: HMM-Based Emotion Detection In Voicemail Messages, Proceedings of the 10th International Conference on Intelligent User Interfaces (IUI '05), San Diego, ACM (2005) 251-253

[7] Salway, A. and Graham, M.: Extracting Information about Emotions in Films, Proceedings of ACM Multimedia, Berkeley, ACM (2003) 299-302

[8] Russell, J., Mehrabian, A.: Evidence for a Three-Factor Theory of Emotions, Journal of Research in Personality, 11 (1977) 273-294

[9] Bradley, M. M.: Emotional Memory: A Dimensional Analysis. In: van Groot, S., van de Poll, N.E., Sargeant, J. (eds.) The Emotions: Essays on Emotion Theory, Hillsdale, NJ: Erlbaum (1994) 97-134

[10] Dietz, R., Lang, A.: Affective Agents: Effects of Agent Affect on Arousal, Attention, Liking and Learning, Proceedings of the Third International Cognitive Technology Conference, San Francisco (1999)

[11] Ortony, A, Clore, G.L., Coolins, A.: The Cognitive Structure of Emotions, Cambridge University Press (1988)

[12] Picard, R: Affective Computing, MIT Press (1997) 


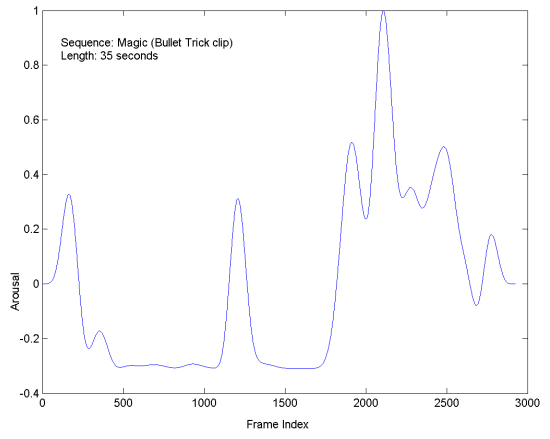

Fig. 1. Magic Bullet Trick: Arousal Graph.

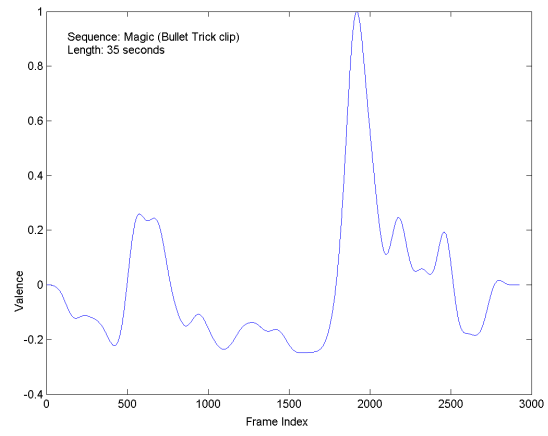

Fig. 2. Magic Bullet Trick: Valence Graph.

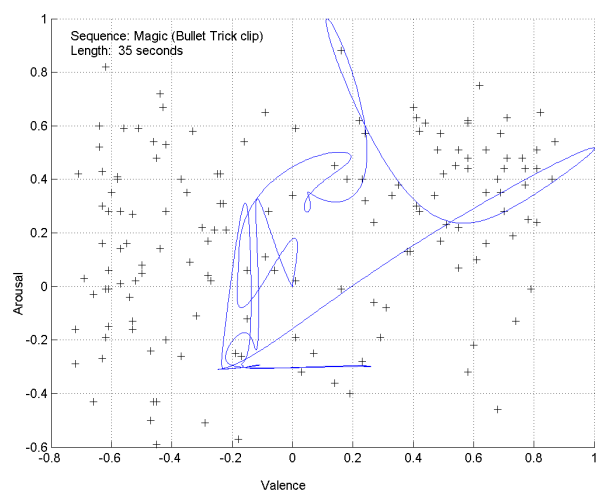

Fig. 3. Magic Bullet Trick: Affect Curve.

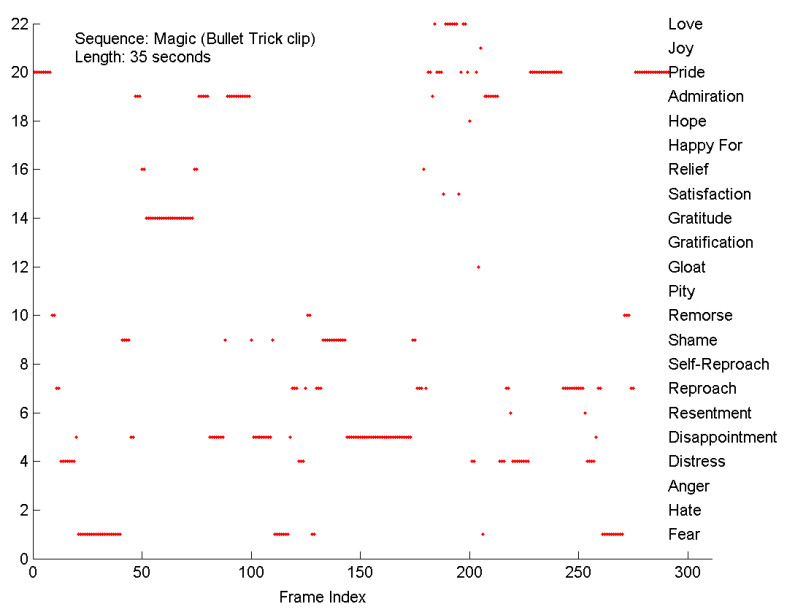

Fig. 4. Magic Bullet Trick: Affect Label Plot. 


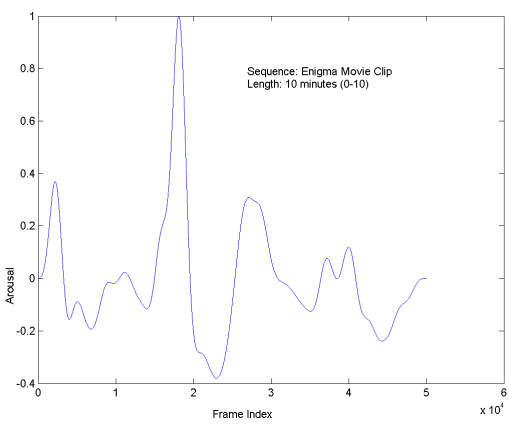

Fig. 5. Enigma 0: Arousal Graph.

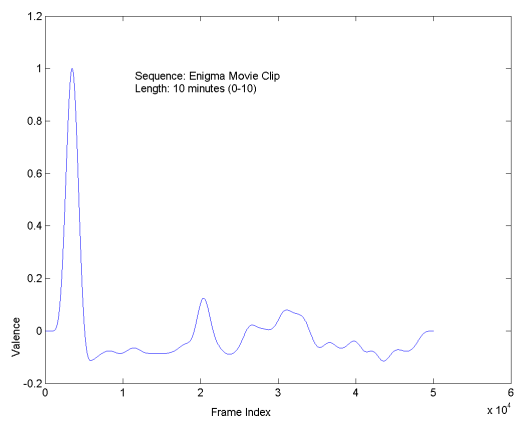

Fig. 6. Enigma 0: Valence Graph.

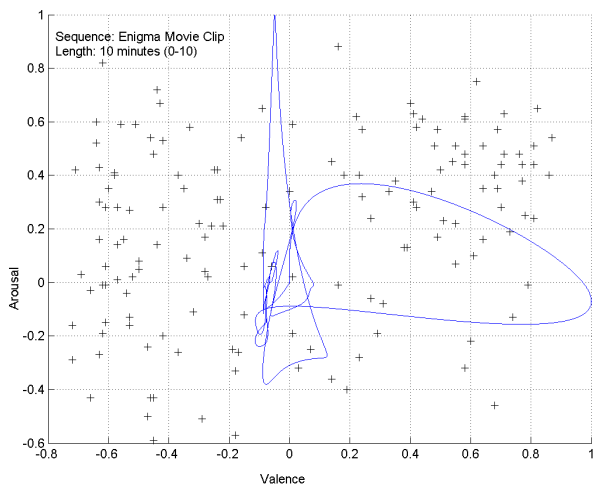

Fig. 7. Enigma 0: Affect Curve.

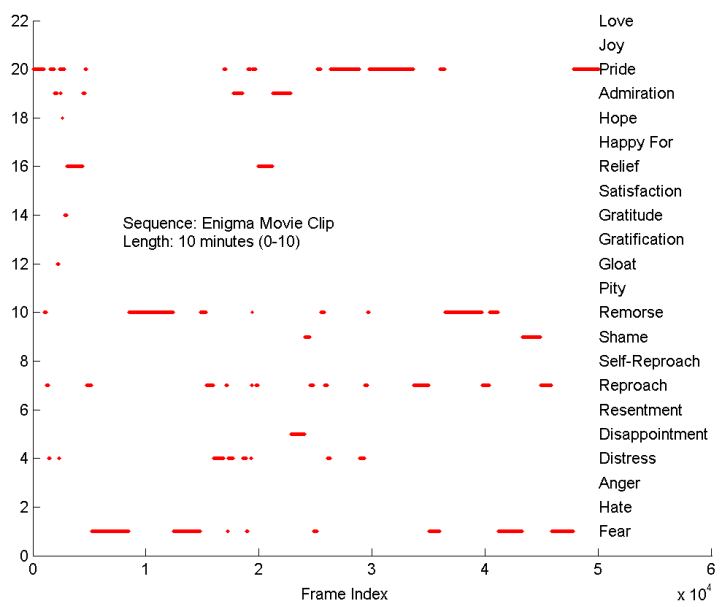

Fig. 8. Enigma 0: Affect Label Plot. 


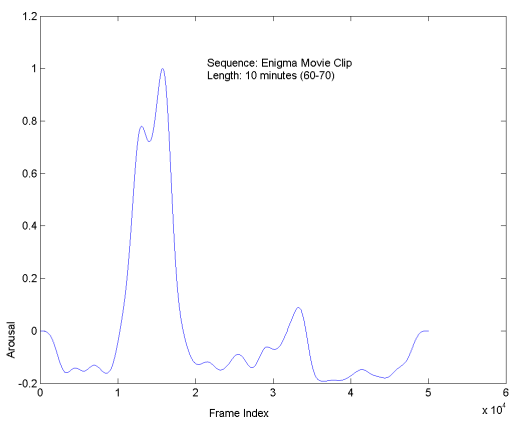

Fig. 9. Enigma 60: Arousal Graph.

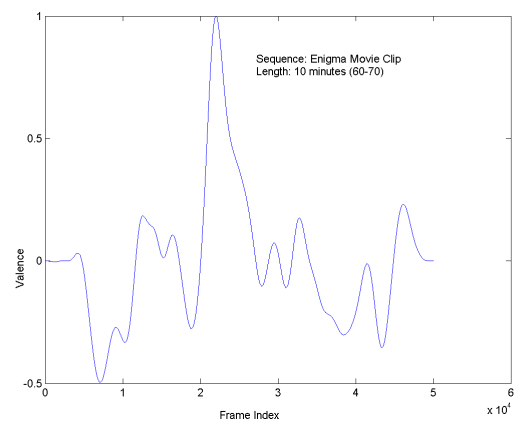

Fig. 10. Enigma 60: Valence Graph.

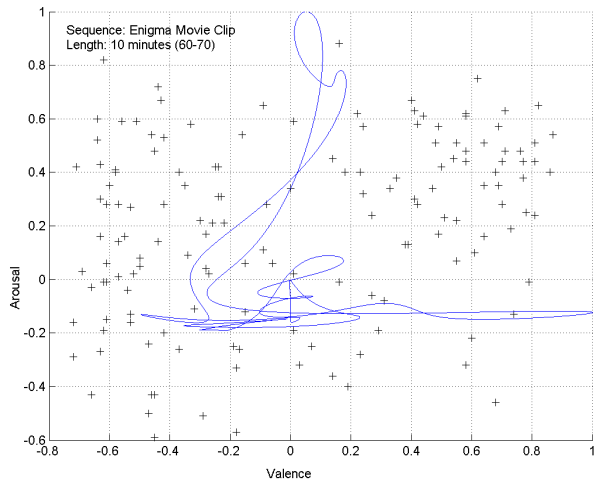

Fig. 11. Enigma 60: Affect Curve.

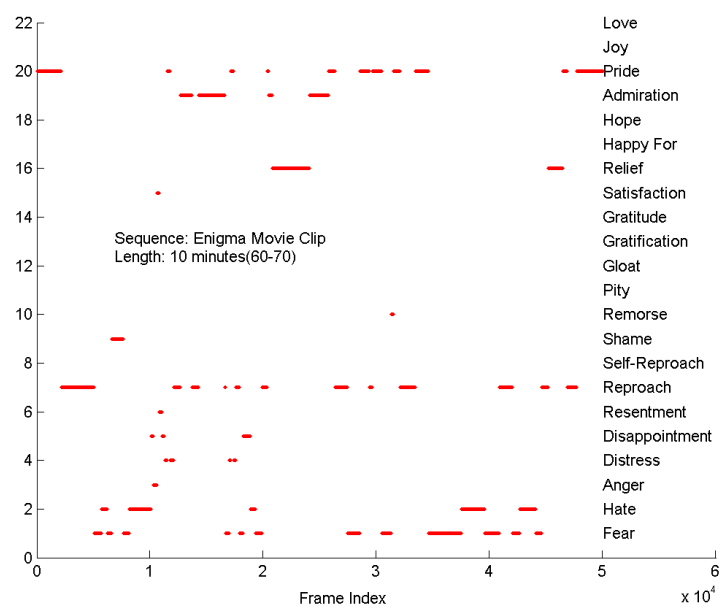

Fig. 12. Enigma 60: Affect Label Plot. 